\title{
Ũber die Konstitution des Urinporphyrins. Gewinnung einer carboxylierten Hämatinsäure aus Urinporphyrin.
}

\author{
Von \\ Hans Fischer. \\ (Ans dem Institut für angew. med. Chemio Innsbruck.) \\ (Der Redaktion zugegangen am 7. September 1916.)
}

Wie schon in früheren Mitteilungen gezeigt wurde, ist das Urinporphyrin ein 4 fach carboxyliertes Kotporphyrin. Es war von Interesse, festzustellen, ob dieser Befund durch den chemischen Abbau des Urinporphyrins gestützt wird und ob Anhaltspunkte für den Ort des Eintritts der Carboxylgruppen gewonnen werden konnten. Dies ist in der Tat gelungen. Es wurde ein 4 fachgechlortes Urinporphyrin gefunden, wodurch im Verein mit der Auffindung des Tetrachlorkotporphyrins ${ }^{1}$ ) bewiesen ist, daß der Eintritt der Carboxylgruppen nicht an den gechlorten Stellen erfolgt sein kann, somit Urin- und Kotporphyrin in ihrer Konstitution in bezug auf diese Stellen übereinstimmen müssen. Es liegt nahe und ist sehr wahrscheinlich, daß es wie im Blutfarbstoff $4 \alpha$-ständige Methingruppen sind, an denen das Halogen substituiert.

Für den Eintritt der Carboxylgruppen blieben hiernach nur $\beta$-Atomgruppierungen übrig und der direkte Beweis für den Eintritt von 2 Carboxylgruppen in $\beta$-Stellung ist auch geglückt. Durch Oxydation des Urinporphyrins wurde eine einfach in $\beta$-Stellung carboxylierte Hämatinsäure gewonnen. $\mathrm{Daß}$ es sich um eine einfach carboxylierte Hämatinsäure handelt, wurde bewiesen durch die Analyse und den Abbau, der sowohl Hämatinsäure als auch Äthylmethylmaleinimid liefert. Es kommen somit für die Konstitution der carboxylierten Hämatinsäure nur folgende 3 Möglichkeiten in Betracht:

1) Diese Zeitschrift, Bd. 98. 


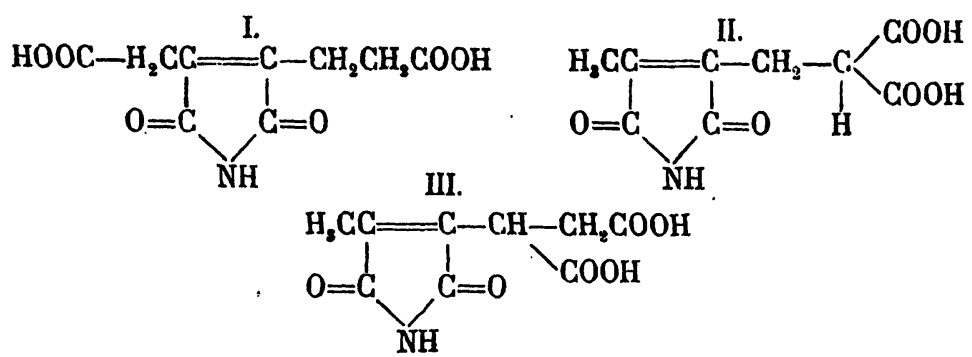

Eine Entscheidung wird wohl kaum anders als auf dem Wege der Synthese erbracht werden können, die ich bei Gelegenheit auszuführen beabsichtige.

Die Ausbeute an krystallisiertem Material übersteigt ein Molekül, wie ja auch die Resultate der oxydativen und reduktiven Spaltung des Kotporphyrins für das Vorhandensein von 2 «sauren, Molekülen in den natürlichen Porphyrinen sprechen. Somit ist der Beweis für die Bindungsart von 2 bezw. 4 Carboxylgruppen im Urinporphyrin erbracht. Daß es sich nicht etwa um eine sekundäre Entstehung von Carboxylgruppen bei der Oxydation handelt, geht daraus hervor, daß der Urinporphyrinester beim oxydativen Abbau zunächst den Ester der carboxylierten Säure liefert, der dann durch Verseifung die freie Säure gibt.

Die totale Reduktion des Urinporphyrins wurde ebenfalls ausgeführt und ergab in Übereinstimmung mit der schon früher beschriebenen des Kotporphyrins keinerlei Basen. In der Säurefraktion konnte keine Phonopyrrolcarbonsäure gefunden werden, ein Verhalten, das jetzt durchaus verständlich erscheint, weil eben durch die relativ kurze Einwirkung des Jodwasserstoffs die Carboxylgruppen nicht abgespalten werden können. Die Pyrrolsäure, die in guter Ausbeute entsteht, ist daher (vgl. den experimentellen Teil) zweifellos eine carboxylierte Phonopyrrolcarbonsäure. Zur Isolierung bezw. Reindarstellung ist natürlich eine größere Menge Ausgangsmaterial notwendig, als mir zur Verfügung stand, zumal da die Gewinnung eines Pikrates mißlang und es nach den bisherigen Erfahrungen bei den Pyrrolsäuren äußerst unwahrscheinlich ist, daß eine 2 fach carboxylierte Pyrrolsäure ein Pikrat liefert. Auch die Aufspaltung mittels Kaliummethylat lieferte ein negatives Resultat, insbesondere 
konnte Phyllopyrrolcarbonsäure nicht nachgewiesen werden, ein Ergebnis, das im Einklang steht mit der festen Bindung der Carboxylgruppen. Da das Kotporphyrin die Phyllopyrrolcarbonsäure in guter Ausbeute liefert, folgt bieraus mit großer Wahrscheinlichkeit, daß die dritte Carboxylgruppe dieses und damit auch eine weitere ( 5 te) des Urinporphyrins im basischen Anteil, und zwar in $\beta$-Stellung, zu suchen ist, wie auch jedenfalls die sechste und siebente des Urinporphyrins unter Berücksichtigung des Befundes der Tetrachlorverbindung.

Diese Anhäufung der Carboxylgruppen im basischen Anteil (hierzu kommen noch die beiden Sauerstoffatome, die vermutlich als Hydoxylgruppen vorhanden sind) bewirkt es offenbar, daß der Basenanteil so labil wird, daß er nur unter besonderen Bedingungen, die ich bis jetzt nicht getroffen habe, isoliert werden kann.

Endlich teile ich eine neue Trennungsmethode von Urinund Kotporphyrin mit, die auf der Schwerlöslichkeit des salzsauren Salzes des Esters des Urinporphyrins beruht.

Tetrachlor-Urinporphyrinmethylester-dihydrochlorid.

$0,1 \mathrm{~g}$ Urinporphyrinmethylester wurden mit Hilfe von $4 \mathrm{ccm}$ Eisessig und $3 \mathrm{ccm}$ konzentrierter Salzsäure in Lösung gebracht und hierzu $1 \mathrm{ccm}$ Wasserstoffsuperoxyd med. zugegeben. Innerhalb 10 Minuten erfolgte der Umschwung nach Grün, dann wurde noch eine Stunde stehen gelassen und jetzt durch vorsichtigen Wasserzusatz der neue Farbstoff in mikroskopischen, radiär gestreiften Kügelchen abgeschieden. Der Körper ist in Chloroform so gut wie unlöslich, weshalb mit diesem Lösungsmittel ausgewaschen wurde, um etwa vorhandenen unveränderten Urinporphyrinester, der in Chloroform spielend löslich ist, zu entfernen. Spektroskopisch waren in der (schwach grün) gefärbten Chloroformlösung ein Streifen im Rot und einer auf der Grenze zwischen Blau und Violett erkennbar. Da sich die Substanz nicht umkrystallisieren ließ, wurde direkt das Rohprodukt analysiert. Wie aus den Analysen hervorgeht, besonders den zu niedrigen $\mathrm{C}$ - und Methoxylwerten, ist in geringem Maße Verseifung der Estergruppen eingetreten, 
indessen kann es keinem Zweifel unterliegen, daß die in der Überschrift genannte Verbindung vorliegt. Auch ist die Substanz hygroskopisch, was ebenfalls die Analysen ${ }^{1}$ ) erschwerte.

$$
\begin{aligned}
& 4,280 \mathrm{mg} \text { Substanz }: 7,66 \mathrm{mg} \mathrm{CO} ; 1,84 \mathrm{mg} \mathrm{H}_{2} \mathrm{O} \\
& 4,960 ;: 3,635>\mathrm{AgCl} \\
& 3,745, \quad: \quad: 3,66: \mathrm{AgJ} \\
& 5,076>\quad: \quad 0,239 \mathrm{ccm} \mathrm{N} \mathrm{N}_{2} \text { bei } 21^{\circ} \text { u. } 701 \mathrm{~mm} \mathrm{Hg} . \\
& \mathrm{C}_{47} \mathrm{H}_{48} \mathrm{~N}_{4} \mathrm{O}_{16} \mathrm{Cl}_{6} \mathrm{Mgw}=1137,18 .
\end{aligned}
$$

$$
\begin{array}{rlll}
\text { Ber.: } \mathrm{C}=49,60 & \mathrm{H}=4,25 & \mathrm{~N}=4,93 & \mathrm{Cl}=18,71 \\
\text { Gef.: } \mathrm{C}=48,81 & \mathrm{H}=4,81 & \mathrm{~N}=5,04 & \mathrm{Cl}=18,13 \\
\text { OCH }_{3} & \text { Ber. : } 19,08 & \text { Gef.: } 16,06 .
\end{array}
$$

Oxydation von Urinporphyrinmethylester.

$1 \mathrm{~g}$ Urinporphyrinmethylester wurde in $25 \mathrm{ccm} 50 \%$ iger Schwefelsäure gelöst, $25 \mathrm{ccm}$ Wasser zugegeben und dann unter Rühren $20 \mathrm{~g}$ Bleidioxyd innerhalb einer halben. Stunde eingetragen. Dabei trat langsam Entfärbung ein; nach 3 Stunden wurde vom Bleidioxyd abgesaugt, mit Wasser nachgewaschen und das Filtrat viermal ausgeäthert.

Der Äther wurde im Vakuum eingedampft, wobei ein Sirup hinterblieb; der auch nach längerem Stehen nicht krystallisierte. Dieses Resultat war nicht weiter verwunderlich, da kein Grund vorhanden war, daß die Estergruppen der zu erwartenden Hämatinsäure verseift seien, hatten doch Küster und Greiner ${ }^{1}$ ) bei der Oxydation des Dimethylhämins gezeigt, daß man so den Methylester der Hämatinsäure erhält, wodurch sie eben den Nachweis der Carboxylgruppen im Hämin erbrachten. Der Rückstand wurde daher nach dem Vorgang der genannten Autoren mit 10\% iger Schwefelsäure verseift, wieder ausgeäthert und der Äther im Vakuum eingedampft. Bemerkenswerterweise trat nun sofort, ohne Animpfen, Krystallisation ein, ein Verhalten, das die Hämatinsäure gewöhnlich nicht zeigt. Nadelförmige Prismen, in Äther relatir schwer löslich, wodurch die Isolierung gelang, wenn auch nur in zu einer Schmelzpunktsbestimmung ausireichenden Menge.

1) Diese hat Herr Dr. Lieb in Graz nach den Preglschen Methoden ausgeführt.

1) Ber., Bd. 45, S. 2503. 
F. P. $175-180^{\circ}$; es liegt also sicher keine Hämatinsäure vor, die bei $114^{\circ}$ schmilzt.

Die Ätherlösung wurde auf ein geringes Volumen gebracht und mit Petroläther versetzt. Alsbald entstand eine Trübung und Abscheidung einer nicht deutlich krystallisierten Substanz (mikroskopisch Kugeln) F. P. $170^{\circ}$, nachdem bei $160^{\circ}$ Erweichen cingetreten war.

4,23j mg Sbst. : $0,218 \mathrm{ccm} \mathrm{N}_{2}$ bei $17^{\circ}$ u. $724 \mathrm{~mm} \mathrm{Hg}$ $=5,77 \% \mathrm{~N}$.

Zur Kohlenwasserstoffbestimmung reichte die erhaltene Menge nicht mehr aus, auch habe ich die Bleidioxydoxydation nicht mehr wiederholt, weil die mit Chromsäure eine viel bessere Ausbeute an dem neuen Körper gab, nachdem die Oxydation direkt mit dem freien Porphyrin ausgeführt wurde.

Oxydation von freiem Urinporphyrin mit Chromsäure.

Ein Gramm freies Urinporphyrin wurden in $25 \mathrm{ccm}$ $50 \%$ iger Schwefelsäure gelöst. Hierzu wurden zwei Gramm Chromsäure in Wasser gelöst langsam unter heftigem Rühren eingetropft. Die Oxydation erfolgte bei Zimmertemperatur fast momentan, bei Anwendung von Eiskühlung schied sich zuerst ein schwer lösliches Chromat ab. Für den Verlauf der Oxydation scheint es gleichgültig $\mathrm{zu}$ sein, ob man bei niedriger Temperatur oder Zimmertemperatur arbeitet.

Nach $4-5$ stündigem Stehen wurde die tiefgrüngefärbte Flüssigkeit $5 \mathrm{mal}$ ausgeäthert und der Ätherauszug in üblicher Weise in eine saure und in eine indifferente Fraktion geschieden. Die indifferente Fraktion war geringfügig, eine krystallisierte Substanz konnte nicht nachgewiesen werden.

Die Rohausbeute an saurer Fraktion wog $0,5 \mathrm{~g}$ und war zum größten Teil schön krystallisiert. Durch Lösen in Aceton, Zusatz von Äther und Eindunsten wurde die Substanz umkrystallisiert und in derben Prismen erhalten. Diese wurden abgesaugt, abgepreßt und zweimal mit absol. Äther ausgewaschen. Die Ausbeute betrug 0,25g, der Schmelzpunkt lag bei 180-183. In den üblichen Lösungsmitteln außer Aceton ist die Substanz recht schwer löslich. $\mathrm{Zu}$ den Analysen wurde bei $100^{\circ} \mathrm{im}$ 
Vakuum über Phosphorpentoxyd getrocknet. $50 \mathrm{mg}$ nahmen hierbei nahezu ein halbes Milligramm ab, und die Substanz schmolz jetzt erst bei $188^{\circ}$ unter Aufschäumen, nachdem ab $170^{\circ}$ geringes Sintern eingetreten war. Für die Ausführungen der Mikro-Kohlenwasserstoffbestimmungen bin ich Herrn Dr. Hans Lieb, der sie nach der Preglschen Methode ausgeführt hat, zu großem Dank verpflichtet.

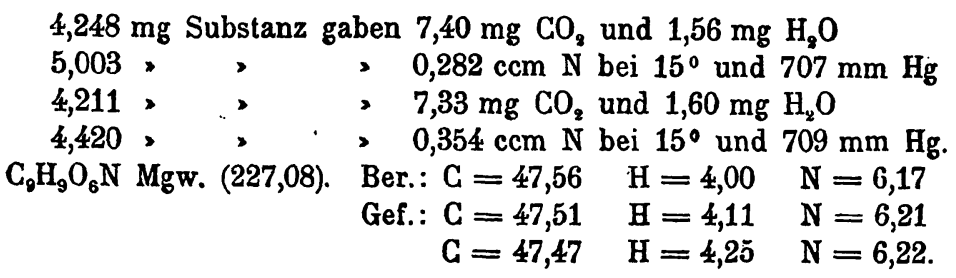

Titration : 0,0102 g Substanz, in Wasser heiß gelöst, verbrauchten $1 \mathrm{ccm} \mathrm{n}$-Natronlauge zur Neutralisation. (Indikator Phenolphthalein); 0,0112 g Substanz, in Alkohol gelöst, verbrauchten $1,15 \mathrm{ccm}$ n-Lauge. (Indikator Phenolphthalein:)

Das sind Werte, die höher als die für eine zweibasische Säure sind. Jedoch steht dies Verhalten im Einklang mit dem der Hämatinsäure, die bei der Titration mit n-Natronlauge auch mehr als ein Molekül bindet.

Abbau der carboxylierten Hämatinsäure.

0,15 g Substanz wurden im Vakuum im Ölbad erhitzt. $\mathrm{Ab} 180^{\circ}$ tritt Sublimation einer Substanz in derben Prismen auf. Es wurde weiter erhitzt bis $220^{\circ}$, ohne daß eine nennenswerte Vermehrung des Sublimats eintrat. Der Rückstand krystallisierte wieder und schmolz nach dem Umkrystallisieren aus Aceton-Äther bei $184^{\circ}$, ist also offenbar unverändertes Ausgangsmaterial. Die sublimierten derben Prismen schmolzen bei $114^{\circ}$ wie Hämatinsäure und die krystallographische Messung von Herrn Dr. Steinmetz in München, dem ich auch hier herzlichst danke, ergab folgendes Resultat: $₫ 1$. Das dünne Röhrchen enthielt einige Kryställchen von undeutlich entwickelten Flächen, ein einziges ließ jedoch die Messung einer Prismenzone $z u$, in der $z$ wischen den besten Flächen ein Winkel von 120 bezw. $60^{\circ}$ gefunden wurde. Am Hämatinsäure-Imid 
$\mathrm{C}_{g} \mathrm{H}_{9} \mathrm{NO}_{4}$ ist der Winkel (130):(100) von Wülfing zu $60^{\circ} 8$, bestimmt worden, soda $B$ im Verein mit dem übereinstimmenden Schmelzpunkt der beiden Substanzen auch der krystallographische Befund mit der Identität beider Substanzen in Einklang steht. Zur optischen Bestimmung war das vorliegende Material leider ungeeignets.

$50 \mathrm{mg}$ Substanz wurden im Reagenzrohr bei gewöhnlichem Druck 3 Minuten lang auf $260^{\circ}$ erhitzt, wobei intensiver Geruch nach Methyläthylmaleinimid auftrat. Der größte Teil der Substanz jedoch blieb als Öl am Boden des Reagierglases, während ein geringer Teil nach oben destillierte und dort krystallisierte. Die Ausbeute war zu gering, als daB auf chemischem Wege eine Identifikation möglich gewesen wäre, weshalb ich das Röhrchen an Herrn Dr. Steinmetz in München sandte, der die Identität mit Methyläthylmaleinimid auf krystallographischem Wege nachwies. Herr Dr. Steinmetz schreibt: «In dem mit soben und sunten gekennzeichneten Röhrchen befindet sich ein durchaus einheitliches Sublimat. Charakteristisch darin sind rechtwinklig knieförmige Zwillingskryställchen, $\square$ deren Zwillingsgrenze unter $4 \check{0}^{\circ}$ gegen die Begren- zungskanten verläuft. Zwischen gekreuzten Nikols löschen diese Zwillinge vollkommen einheitlich parallel den rechtwinkligen Kanten aus, bei Anwendung 1/4 Undulationsplatte zeigt der Farbenunterschied der beiden Einzelkryställchen aber deren verschiedene optische Orientierung an. Ganz ähnliche Zwillinge zeigte ein Vergleichspräparat von subl. Methyläthylmaleinimid. Die Erklärung dieses eigentümlichen Verhaltens ergibt sich aus der früher von mir ausgeführten Messung der Substanz; sie krystallisiert rhombisch, und der Winkel der Fläche (011) zu (010) beträgt $44^{\circ}$ 57', also nahezu $45^{\circ}$. Tritt nun nach (011) Zwillingsbildung ein, so müssen Gebilde von den erwähnten optischen Eigenschaften entstehen, da die kleine Differenz von $3^{\prime}$ bei so kleinen Krystallen, wie die vorliegenden waren, nicht mehr mit Sicherheit festgestellt werden kann, also praktisch verschwindet.

Die Identität des fraglichen Präparates mit dem Methyläthylmaleinimid kann daher als ganz sicher bezeichnet werden ». 
Die Oxydation des Urinporphyrinmethylesters wurde unter ähnlichen Bedingungen ausgeführt wie die mit Bleidioxyd. Zunächst wurde der Ester der carboxylierten Hämatinsäure gewonnen, aus dem durch Verseifen mit Schwefelsäure in der oben beschriebenen Weise die freie Säure erhalten wurde. Ausbeute $78 \mathrm{mg} ;$ Schmelzpunkt $183^{\circ}$. Durch die Schwefelsäure muß jedoch noch eine Veränderung sekundärer Arl erfolgt sein, da die Analysen im Kohlenstoff einen um 0,5\% zu hohen Wert ergaben.

Totale Reduktion des Urinporphyrins.

In der ersten Mitteilung über das Urinporphyrin ${ }^{1}$ ) ist schon einmal die totale Reduktion dieses Farbstoffes beschrieben worden. Es wurde festgestellt, daß die Basenfraktion fehlt, und in der Säurefraktion wurde eine geringe Menge Phonopyrrolcarbonsäure als Pikrat isoliert. Gegen den Ausfall dieses Versuches konnte mit Recht der Einwand erhoben werden, daß er mit dem Rohprodukt ausgeführt wurde, ich habe daher nochmals einen Kontrollversuch mit reinem krystallisierten Ester ausgeführt.

1. c. ist zwar schon ein Kontrollversuch mit $0,6 \mathrm{~g}$ reinem krystallisierten Material ausgeführt worden und das Resultat in bezug auf das Fehlen der Basenfraktion vollkommen bestätigt worden. Phonopyrrolcarbonsäure konnte nicht als Pikrat isoliert werden, jedoch wurde diesem negativen Ergebnis in Anbetracht der geringen Menge Ausgangsmaterial bei der gegenüber den Basen viel schwieriger nachweisbaren Phonopyrrolcarbonsäure keine entscheidende Bedeutung beigelegt; sehr mit Unrecht, wie der nachstehende Versuch zeigt.

$2 \mathrm{~g}$ Urinporphyrinmethylester wurden mit $20 \mathrm{ccm}$ Eisessig und $10 \mathrm{ccm}$ Jodwasserstoffsäure (spez. Gewicht 1,96) 2 Stunden lang im siedenden Wasserbad erhitzt, das Jod mit Jodphosphonium reduziert, dann das Lösungsmittel im Vakuum abdestilliert und der Rückstand mit Wasser und Sodalösung aufgenommen. Der Ätherextrakt hiervon gab keine Ehrlichsche Reaktion in Be-

1) H. Fischer, Diese Zeitschr., Bd. 95, S. 58. 
stätigung der früheren Versuche, bei denen schon das Fehlen der Basenfraktion festgestellt wurde.

Die Sodalösung wurde jetzt eben bis zur schwärzlichen Verfärbung von rotem Kongopapier mit Schwefelsäure angesäuert und viermal mit Äther ausgezogen. Der Ätherextrakt wurde im Wasserbad unter stark vermindertem Druck eingedampft und zuletzt noch 2-3 Minuten im siedendem Wasserbad erhitzt, um Reste von Essigsäure zu entfernen. Beim Stehen über Nacht trat keine Krystallisation ein. (Vgl. dagegen den früher beschriebenen Reduktionsversuch von Kotporphyrinmethylester.) Dabei war die vorhandene Menge nicht unbeträchtlich, sie wog $0,7 \mathrm{~g}$ und löste sich spielend in heißem Wasser im Gegensatz zu den Säurefraktionen von Blut- und Gallenfarbstoff.

Hiernach konnte keine Phonopyrrolcarbonsäure vorliegen, jedoch bestanden die $0,7 \mathrm{~g}$ sicher der Hauptsache nach aus Pyrrolsäuren, da die Aldehydreaktion intensiv positiv war.

Nun wurde mit Methylalkohol und trockener Salzsäure in üblicher Weise verestert und die Ester nach $F$ ischer und Rös ${ }^{1}$ ) verarbeitet. In einer Probe wurde zunächst die Isolierung des charakteristischen Phonopyrrolcarbonsäurepikrats versucht, ohne Erfolg, und dann die entstandenen Ester im Vakuum destilliert.

Fischer und Röse hatten festgestellt, daß die Säureester des Blutfarbstoffs bei $220^{\circ}$ Ölbadtemperatur vollkommen überdestillieren, hier dagegen trat erst bei $230^{\circ}$ Ölbadtemperatur Destillation ein. (Es ist klar, daß beim Vorliegen solch geringer Mengen nur die Ölbadtemperatur maßgebend sein kann.) Auch im farblosen Destillat, das die Ehrlichsche Probe intensiv gab, konnte kein krystallisiertes Pikrat nachgewiesen werden.

Durch den Ausfall dieses Versuches halte ich das Nichtentstehen von Phonopyrrolcarbonsäure für erwiesen.

Diese Versuche wurden ausgeführt, ehe die Oxydation des Urinporphyrins vorgenommen war. Ich zweifle nicht daran, daß, wenn man, anstatt der Ester der Pyrrolsäuren wie oben beschrieben, die freien Säuren destilliert, mit ziemlicher Sicherheit Phonopyrrolcarbonsäure erhalten wird. Ebenso wird man

1) Ber. d. Dtsch. Chem. Ges., Bd. 47, S. 792. 
sehr wahrscheinlich aus Urinporphyrin bei 16-17stündigem Kochen mit Eisessig-Jodwasserstoff Phonopyrrolcarbonsäure unter Kohlensäureabspaltung erhalten. Aus Mangel an Material habe ich die beiden wichtigen Versuche nicht ausführen können.

Verhalten von Urinporphyrinmethylester gegen Kaliummethylat.

In völlig analoger Weise wie früher der Kotporphyrinester wurde Urinporphyrinmethylester mit Kaliummethylat bebandelt. Die Temperatur- und Druckkurve stimmt mit der angegebenen völlig überein. Der Inhalt des Silberbechers war grün, es war also keine totale Aufspaltung eingetreten.

Mit Wasserdampf wurde ein rapid sich grün färbendes, ursprünglich farbloses Öl abgetrieben, aber in so geringer Menge, daß eine Charakterisierung nicht möglich war.

In der Säurenfraktion konnte Phyllopyrrolcarbonsäure nicht nachgewiesen werden.

Neue Trennungsmethode von Urin-und Kotporphyrin. ${ }^{1}$ )

Durch Essigsäure wird aus Porphyrinurin ein Gemisch von Urin- und Kotporphyrin niedergeschlagen, das man sehr einfach in folgender Weise trennen kann: 2,5 $\mathrm{g}$ Rohporphyrinfällung wird mit $30 \mathrm{ccm}$ Methylalkohol, der mit trockener Salzsäure gesättigt ist, übergossen und die Flüssigkeit öfters umgeschwenkt. Nach 24 Stunden, manchmal dauert es auch etwas länger, krystallisiert der Urinporphyrinester, bezw. sein salzsaures Salz in glänzenden, derben Prismen aus, die man absaugt und mit Methylalkohol auswäscht. Mit Bicarbonatlösung wird dann das salzsaure Salz zerlegt, der Ester in Chloroform übergeführt und dann durch Zugabe von heißem Alkohol in üblicher Weise zur Krystallisation

1) Herrn Dr. Schumm in Hamburg, dem ich denPorphyrinpatienten Petry gelegentlich meiner Übersiedelung nach Innsbruck zur weiteren wissenschaftlichen Untersuchung anbot, bin ich zu Dank verpflichtet dafür, daß er dem Patienten erlaubt hat, mir auch weiterhin einen Teil des Rohporphyrins seines Urins sowie seinen Kot, soweit sie in Hamburg nicht benötigt werden, zuzusenden.'

Es wäre mir ohne dies Entgegenkommen nicht möglich, die Untersuchung des Urinporphyrins weiter fortzuführen. 
88 Hans Fischer, Über die Konstitution des Urinporphyrins.

gebracht. Die Mutterlauge unterwirft man zweckmäßig nochmals dem gleichen Verfahren, nur dauert dann die Abscheidung der Krystallisation etwas länger; auch ist es jetzt zweckmäBig, in konzentrierterer Lösung $\mathrm{zu}$ arbeiten als oben beschrieben.

In den Mutterlaugen befindet sich das Kotporphyrin noch verunreinigt durch Urinporphyrin. Die Reindarstellung des Kotporphyrins wird durch Behandlung mit Äther in der früher beschriebenen Weise erzielt. Übrigens kann man die Veresterung des Rohporphyrins selbstverständlich auch durch Methylalkohol und Schwefelsäure vornehmen, von letzterer nimmt man zweckmäßig 10\%. In diesem Fall tritt die Abscheidung eines schwerlöslichen Salzes nicht ein und man muB daher nach dem in der ersten und zweiten Mitteilung über die natürlichen Porphyrine angegebenen Verfahren arbeiten. 